\title{
Disambiguating Visual Motion by Form-Motion Interaction-a Computational Model
}

\author{
PIERRE BAYERL AND HEIKO NEUMANN \\ Department of Neural Information Processing, University of Ulm, Germany. \\ \{pierre.bayerl, heiko.neumann\}@uni-ulm.de
}

Received December 6, 2004; Revised July 25, 2005; Accepted February 15, 2006

First online version published in June, 2006

\begin{abstract}
The neural mechanisms underlying motion segregation and integration still remain unclear to a large extent. Local motion estimates often are ambiguous in the lack of form features, such as corners or junctions. Furthermore, even in the presence of such features, local motion estimates may be wrong if they were generated near occlusions or from transparent objects. Here, a neural model of visual motion processing is presented that involves early stages of the cortical dorsal and ventral pathways. We investigate the computational mechanisms of V1-MT feedforward and feedback processing in the perception of coherent shape motion. In particular, we demonstrate how modulatory MT-V1 feedback helps to stabilize localized feature signals at, e.g. corners, and to disambiguate initial flow estimates that signal ambiguous movement due to the aperture problem for single shapes. In cluttered environments with multiple moving objects partial occlusions may occur which, in turn, generate erroneous motion signals at points of overlapping form. Intrinsic-extrinsic region boundaries are indicated by local T-junctions of possibly any orientation and spatial configuration. Such junctions generate strong localized feature tracking signals that inject erroneous motion directions into the integration process. We describe a simple local mechanism of excitatory form-motion interaction that modifies spurious motion cues at T-junctions. In concert with local competitive-cooperative mechanisms of the motion pathway the motion signals are subsequently segregated into coherent representations of moving shapes. Computer simulations demonstrate the competency of the proposed neural model.
\end{abstract}

\section{Introduction}

The mechanisms of motion processing in biological vision systems, in particular the influence of form information on the integration and segregation of local motion estimations, remain largely unclear. Regions with different form configurations can be divided into three classes, which contribute differently to the estimation of local motion (Zetzsche and Barth, 1990; Kalkan et al., 2004): (1) regions with homogeneous intensity are denoted as intrinsic 0-dimensional (i0D) structures, because there is no variation of luminance in any dimension. Such regions remain unchanged even in the presence of visual motion. Hence, such regions do not contribute to the detection of local motion. (2) Luminance variations in regions with contrasts of constant orientation can be described in one dimension (along the orientation of the contrast) and thus are denoted as intrinsic 1-dimensional (i1D) structures. Here, only the part of the true visual motion projected onto the luminance gradient orientation can locally be measured (aperture problem). Thus, the true direction of motion can be constrained but not fully extracted. (3) Regions with contrasts of different orientations are called intrinsic 2-dimensional (i2D) structures, because a full $2 \mathrm{D}$ basis is necessary to describe the local luminance structure. Such localized luminance configurations, or features, often are unique in a certain neighborhood 
which makes it possible to extract the true local visual motion. This measurement, however, does not have to be consistent with the true image motion of the underlying moving object (see below).

The fact that intrinsic 1D structures mostly occur as a consequence of apertures, or spatially limited receptive fields for the analysis of local motion, the problem of ambiguous motion arising at such locations is called the motion aperture problem. Such ambiguities can be resolved by the combination of several local measures of one single moving form, e.g., by the intersectionof-constraints approach (IOC; Adelson and Movshon, 1982). In this approach, measurements from different i1D regions or from $\mathrm{i} 1 \mathrm{D}$ and $\mathrm{i} 2 \mathrm{D}$ regions are combined to compute the true direction of motion (see Fig. 1a,b).

In the presence of multiple objects moving in different directions, it is important to combine only features from same objects, in order not to generate wrong estimations for object motion (see Fig. 1c, inlay A). Consequently, in order to correctly determine object motion, a preceding segmentation of the scene is necessary. On the other hand, object boundaries may also be defined only by the motion signal. Thus, we have a Chicken-and-Egg problem, in which integrated motion cues initiate the segmentation process (motion based segmentation) and, at the same time, using the segmentation of the scene leading to correct motion estimations (form based segmentation). Furthermore, in the presence of occlusions of differently moving objects, localized features (i2D) at T-junctions can lead to erroneous unambiguous motion signals (see Fig. 1c, inlay B; McDermott et al., 2001). In order to identify such occlusions and thus possible erroneous motion cues, form information can be utilized, such as the direction of figure (DOF), which is the direction pointing towards the surface region a certain contour belongs to (Baek and Sajda, 2003).

The presented work is motivated by knowledge about the function of the primate visual system, which is organized in different processing streams or pathways (Van Essen and Gallant, 1994). In the ventral pathway mainly form information is processed, whereas in the dorsal pathway visual motion is analyzed. Psychophysical investigations suggest that motion features are integrated only when they are intrinsic to (or belong to) the moving boundary, while extrinsic signals (i.e. those that belong to a different object surface) are suppressed (Shimojo et al., 1989). We suggest that interactions between the ventral form path and the dorsal motion path are essential for the decision to correctly integrate and segregate visual motion.

The problem we address in this paper is the correct interpretation of local motion estimations. On one hand, estimations belonging to individual objects have to be combined and not mixed up with estimations belonging to different objects. On the other hand, erroneous but unambiguous motion estimations occurring at occlusions must not be assigned to any object. Several models which have been proposed to solve this problem have in common, that they make use of global motion information or need complex processing of form information before motion can be integrated.

Sajda and Baek (2004) proposed a model, in which border ownership indicated by the direction of figure (DOF) is used to steer the integration process: First, the form information is processed and the DOF extracted. Second, the relaxed model state of the form path modulates the input to the motion path. Third and finally, belief propagation is utilized to combine motion information along contours. This information, in turn, is interpreted in a global context, combining relaxed motion information from different contours in the scene. A drawback of this model is the strict sequential processing of form and motion information which stands in contradiction with the dynamic nature of motion signals. In addition, its function may be questioned since the described interactions are not demonstrated to work in a full dynamic system. Furthermore, the authors focus on the integration of global motion and do not investigate how local cues can be segregated, e.g. in the presence of multiple moving objects.

Weiss and Adelson (1994) introduced a model which combines concepts of perceptual organization (integration of information in a spatial neighborhood) with the EM-algorithm (expectation maximization). In addition to the EM algorithm (Dempster et al., 1977; Weiss, 1997) which processes global motion, they realize a local integration of detected likelihoods in a spatial neighborhood by the anisotropic diffusion of extracted motion signals along contours. In order to correctly process image sequences with overlapping objects that cause several erroneous local motion cues, such as for the diamond sequence described in (McDermott et al., 2001; see Fig. 1c), they initially segment a frame of the sequence. The segmentation is utilized to identify motion measurements lying on contours and to bias the spatial integration using contour ownerships to fill in homogeneous regions without motion 


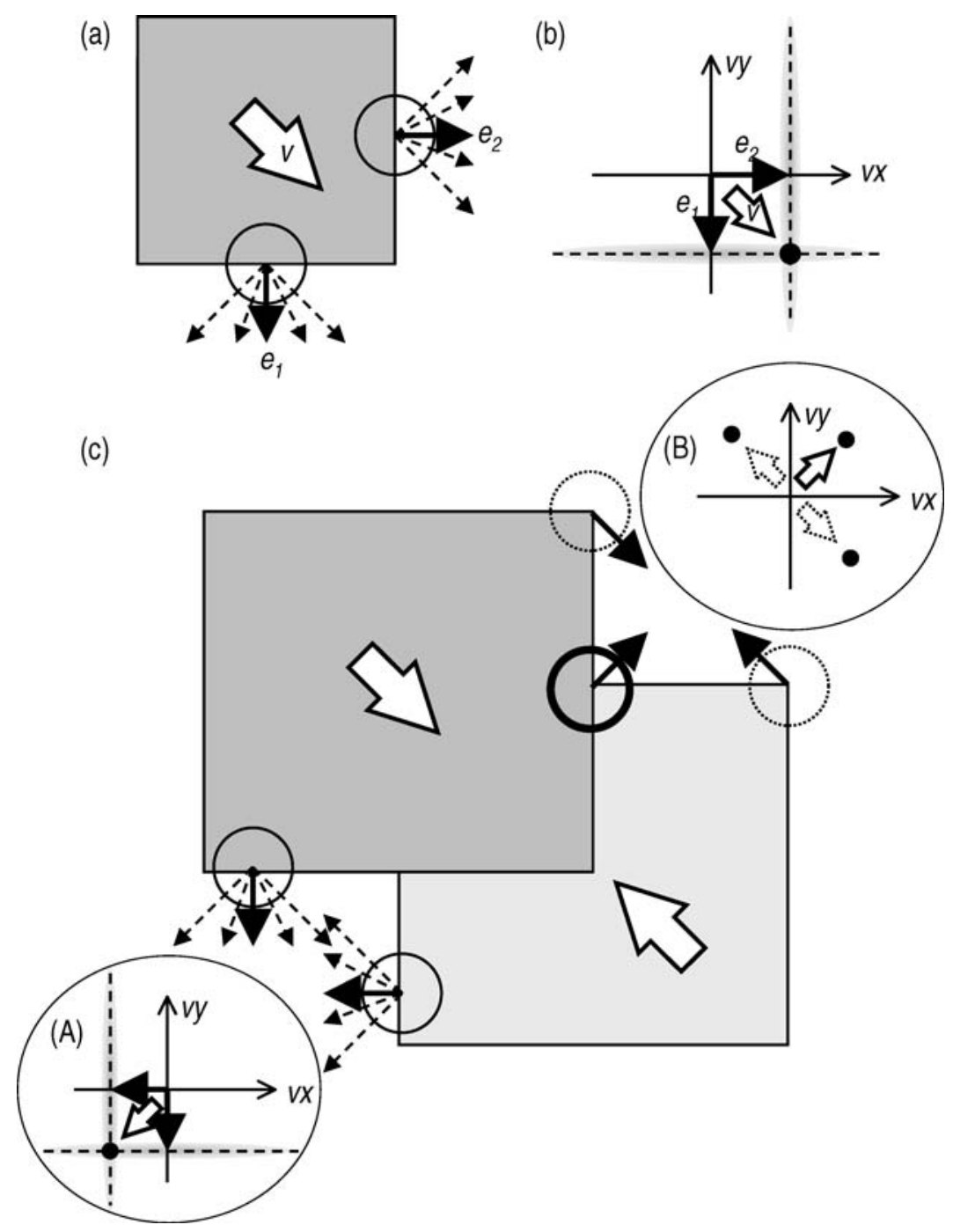

Figure 1. Schematic illustration of the aperture problem, (a) shows ambiguous motion estimations which are detected by an arbitrary local motion detector at two edges of a diagonally moving rectangle. The true image motion is indicated by an outlined arrow. Possible image motion at locations marked with an outlined circle are indicated by arrows: bold arrows $\left(e_{1}, e_{2}\right)$ indicate normal flow and dashed arrows possible hypotheses of the real image motion, (b) In velocity space (vx/vy) the normal flow detected in (a) can be utilized to constrain the set of possible motion configurations by so-called motion constraint lines (shown as dashed lines). The intersection of constraints (IOC) yields the true image motion and can be implemented in a voting scheme (for example by populations of cells which sample the velocity space), (c) demonstrates that object configurations containing occlusions, such as two overlapping moving diamonds (adapted from McDermott et al., 2001), may yield wrong motion cues. Motion cues are illustrated similar as in (a). If motion cues from different objects are combined wrong motion estimations may be generated (inlay A) At junctions unambiguous motion is extracted, but if a junction is formed by an occlusion this unambiguous motion cue may point in the wrong direction (inlay B, bold arrow). Furthermore, the combination of motion features from corners belonging to different objects leads to conflicting configurations, where more than one velocity is indicated (inlay B, dotted arrows).

measurements. Similar to the approach of Sajda and Baek (2004), a complex processing of form information is needed before motion integration may start. Furthermore, the EM-algorithm poses an additional problem that the number of objects to be assumed in the scene has to be determined.
Grossberg et al. (2001) presented a model of motion integration and segmentation in area MT and MST based on dynamic inputs from a figure ground separation process in the form pathway. The authors propose how motion is processed across apertures (which is one of their major contributions, but which is not 
discussed here) and what kind of motion cues arise from form information at occlusions. The key mechanism to correctly handle occluded moving form is based on the preceding form processing, which separates intrinsic/extrinsic form configurations. Thus, no unambiguous erroneous motion features arise at occlusions, such as in Fig. 1c. A drawback of their model is that it does not generate localized motion estimates, since it employs integration ranges covering large parts of their input patterns. Also the authors do not demonstrate that their model is able to correctly handle inputs, which exceed the maximal size of their integration ranges, nor has it been tested on natural image sequences.

In this paper, we investigate possible mechanisms of the motion pathway to locally process visual motion, and propose how these mechanisms are influenced by information from the form pathway. In particular, we analyze the local dynamics of the motion pathway used to generate spatially segregated local motion estimations. Unlike other approaches we do not use an explicit tag denoting the DOF to influence the motion processing and do not use any global operations, such as in the EM-algorithm (Weiss and Adelson, 1994) or the global interpretation of motion using motion estimation from the entire field of view (Sajda and Baek, 2004). Instead, we introduce a form-motion interaction mechanism which uses information about the possible locations of occlusions to steer the local integration and segregation of velocity. This information flow from the ventral form stream to the dorsal motion stream is proposed to be purely excitatory, which in concert with proper competitive mechanisms leads to the desired net dynamical effects. The biologically motivated investigation leads to a model of the functionality and architecture of mechanisms that are involved in motion detection, integration, and segregation. Such principles are also of interest for computer vision to build computational systems that work in domains of possibly unconstrained complexity.

\section{Model}

We extend our previously proposed model of recurrent visual motion processing (Bayerl and Neumann, 2004), which is able to solve the aperture problem in the absence of object occlusions in a temporal sequence of images. This model consists of two cortical model areas, namely V1 and MT, which utilize integration and competition processes on two different spatial scales. The mechanisms that were utilized to describe the model dynamics are the same in both model areas. In the following we briefly review the computational mechanisms of motion integration and segregation in these areas (sketched in Fig. 2, left). Differences between both areas, if any, are explicitly notified. Then we propose a mechanism of formmotion interaction to influence the integration process in the motion pathway. This mechanism utilizes excitatory interaction that aims at providing localized information about occlusions from the form pathway (sketched in Fig. 2, right).

\section{Motion Integration and Segregation}

Three key mechanisms are utilized to integrate and segregate visual motion, which are summarized in this paragraph and subsequently explained in detail in the following paragraphs. First, input information is integrated along the feedforward direction in two subsequent model areas with cells having increasing receptive field sizes. As a consequence, the first model area with cells with small receptive field sizes represents spatially more localized motion information, while the second area with cells with larger receptive field sizes is less affected by the aperture problem and thus indicates more precise velocity estimations by including context information from a spatial neighborhood. Second, shunting inhibition guarantees that the network obeys bounded input-output stability. Third, to finally combine the information that is represented in both model areas, feedback from the second model area modulates the inputs to the first model area in a recurrent loop. Such a reentry of activity from higher processing stages (Sporns et al., 1989) allows selectively biasing input (motion) cues by a soft gating mechanism (Neumann and Sepp, 1999) with information from a larger context (compare attention, biased competition; Desimone and Duncan, 1995).

Excitatory feedback (Hupé et al., 2001) from higher areas is realized by a modulation of the input signal with a value greater or equal to 1 . The modulatory enhancement is defined by a multiplication of the input with the sum of 1 and the feedback signal (Eq. 1, left and center part of equation). This feedback interaction can be rewritten as a sum of the feedforward input and the multiplicative correlation of the feedforward and the feedback signal (Eq. 1, right part of equation). Thus, in the absence of feedback the input signal is left unchanged, while it is enhanced if the feedback signal is in resonance with the input signal (adaptive resonance theory; Grossberg, 1980). The logic behind this 
(a)

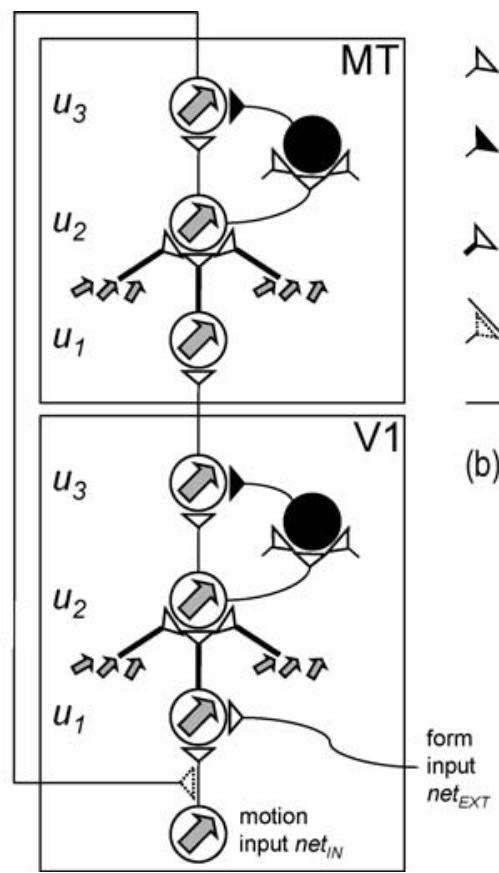

(b) excitatory (additive)

connection

inhibitory (divisive) connection

nonlinear excitatory

(additive) connection

modulatory excitatory

connection
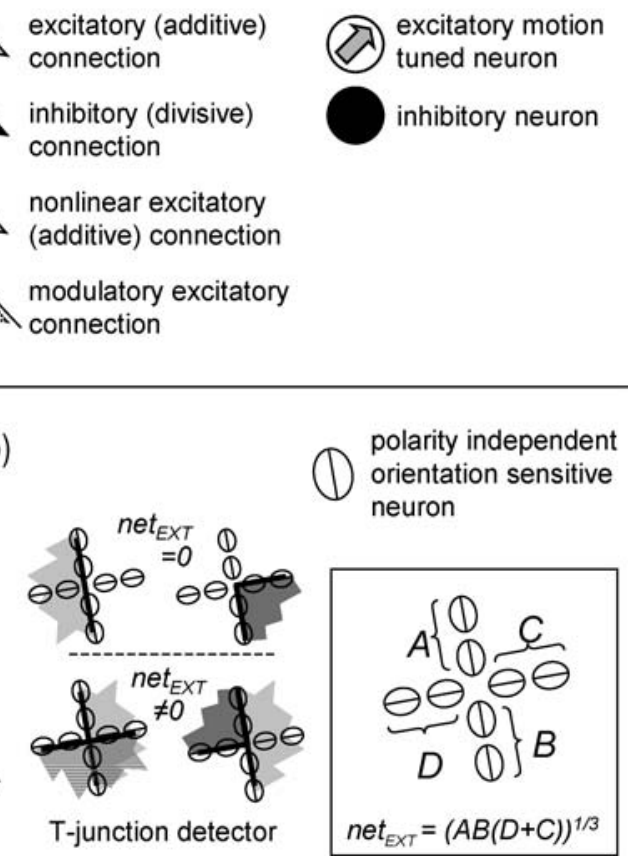

Figure 2. (a) Overview of model areas VI and MT. (b) Computation of net EXT $_{\text {to }}$ to detect possible occlusions using cells sensitive to oriented contrasts: $n^{e t} t_{E X T}=0$ for the elongated contrast and L-junctions; net $_{E X T} \neq 0$ for $\mathrm{X}$ - and T-junctions.

operation is that higher areas encode more context information and thus may bias the input information in earlier areas (Grossberg, 1980; Neumann and Sepp, 1999). As a consequence, biased activity patterns subsequently have a competitive advantage in the next interaction steps of the computation.

In the context of visual motion processing in the presented neural network, motion likelihoods constraining possible detected velocities, are encoded by activity patterns of subpopulations of cells at each location. Hence, the pair wise multiplication of cell activities from two such subpopulations corresponds to the combined constraints of both subpopulations. Thus, the correlation of feedback and input signals can be interpreted as neural implementation of the intersection of constraints approach (IOC; Adelson and Movshon, 1982).

$$
\begin{aligned}
u_{x, v}^{(1)} & =n e t_{I N} \cdot\left(1+C \cdot n e t_{F B}\right) \\
& n e t_{I N}+C \cdot \underbrace{n e t_{I N} \cdot n e t_{F B}}_{\begin{array}{l}
\text { combination of } \\
\text { constraints (see text) }
\end{array}}
\end{aligned}
$$

In Eq. $1, u^{(1)}{ }_{x, v}$ denotes the response of a cell tuned to velocity $v$ at location $x$, net $t_{I N}$ specifies the input signal of each area encoding velocities represented by a population of cells and $n e t_{F B}$ is the feedback signal from higher areas. Both, net $t_{I N}$ and net $_{F B}$ describe scalar values representing neural activity for different velocities $v$ at different locations $x$ similar to $u^{(1)}{ }_{x, v}$, we have omitted the indices $x$ and $v$ for better readability. The input (net $\left.t_{I N}\right)$ to model area V1 is computed by a mechanisms similar to the Extended Reichardt Detector (ERD; Adelson and Bergen, 1985) which correlates oriented complex cell responses ${ }^{1}$ in a neighborhood defined by a Gaussian-shaped receptive field $(\sigma=1.0)^{2}$. The resulting motion information is represented by populations of cells tuned to different velocities $v_{i} \in$ $\{-7,-6, \ldots, 6,7\}^{2}$ pixel/frame at each location in the image. Model cells in area V1 receive feedback from the output of model area MT $(C=100)$. As sketched in Fig. 2 cells in model area MT receive no feedback from any higher order model area $(C=0)$.

The proposed modulatory excitatory feedback interaction has the following advantages in comparison to direct multiplicative gating mechanisms or additive/subtractive feedback: additive excitation could 
produce activation patterns which are not present in the input pattern, while subtractive inhibition could completely suppress the input pattern. Both, additive and subtractive interactions are dependent on the strength of the input signal and, thus, are difficult to parameterize. Such mechanisms implement so-called strongloops which often lead to instabilities in network behavior that cannot be controlled in a working system (Crick and Koch, 1998). Direct modulatory interaction (gating) has the advantage to automatically scale its effect with the strength of the input signal and does not generate activity where no input is present. In contrast to the employed excitatory modulation, direct modulation may suppress relevant information in the input signal in cases where no feedback is present. To overcome this drawback we employ the described mechanism of excitatory modulation (soft-gating; Neumann and Sepp, 1999).

The feedforward integration of the top-down biased signal $u^{(1)}$ is realized by two operations: the signal $u^{(1)}$ is squared to sharpen the response and is then integrated by utilizing isotropic Gaussian filters in the velocity domain ( $\sigma=0.75$ in both model areas) and in the spatial domain (V1: $\sigma=0$; MT: $\sigma=7$ ). The computation is denoted by Eq. 2 :

$$
u_{x, v}^{(2)}=\left(u_{x, v}^{(1)}\right)^{2} * G_{\sigma_{1}}^{(\text {space })} * G_{\sigma_{2}}^{(\text {velocity })}
$$

where "**" represents the convolution operation over the respective domains. The ratio of the spatial extend of retinal receptive fields of model area MT to model V1 is approximately 1:5. Thus, activity patterns in model MT contain more context information and are less affected by the aperture problem. The nonlinear signal transformation realized by the squaring operation has the property to change the relative difference of activities of cells in one subpopulation. This is important for the gradual evolution of a set of winner-neurons to encode the detected velocity.

As last stage of each model area, lateral shunting inhibition is employed to normalize the signal, which guarantees that the activity of the model is kept within predefined bounds which depend on the model parameter setting. The inhibitory term is obtained by summing over all $n$ velocities $v$ at each location. Such an interaction leads to a strong decrease of neural activity in the model at locations where ambiguous signals indicate many possible velocities (Simoncelli and Heeger, 1998), while unambiguous motion signals that indicate only one or a few velocities receive much less inhibition (Eq. 3).

$$
u_{x, v}^{(3)}=\frac{u_{x, v}^{(2)}}{\varepsilon+\sum_{v} u_{x, v}^{(2)}}, \text { with } \varepsilon=0.01
$$

As a consequence of the proposed mechanism, salient motion cues are implicitly detected at line endings or corners, where motion estimations are unambiguous. Along elongated contrasts ambiguous estimations occur as a result of the aperture problem, which indicates motion perpendicular to the contour of an object.

Finally, iterative feedforward/feedback processing is necessary to combine the effect of each of the described mechanisms in both model areas to achieve the desired disambiguation of visual motion estimations. Feedback modulation amplifies model V1 cell activities which cohere with salient activity patterns represented in model MT. Those motion patterns, which are enhanced by the feedback signal, get a competitive advantage in the following processing stages. A consequence is an increase of saliency and less ambiguous motion patterns. The increased receptive field size in model MT leads to a spatial interaction between neighboring locations. Model V1 activity patterns are biased via recurrent modulation by motion patterns from model MT containing information from a larger spatial context. Thus, initiated at line endings or corners, unambiguous or disambiguated information propagates along extended boundaries. The net effect of our model is the filling-in of salient motion features through recurrent motion processing.

\section{Form-Motion Interaction}

In the case where differently moving objects overlap, erroneous motion may be detected even in the presence of localized spatial features (McDermott et al., 2001; compare Fig. 1c). Such unambiguous but erroneous motion cues propagate and stabilize their information through the described mechanisms leading to undesired results. Here we propose a mechanism to incorporate the location of such occlusion cues in the motion disambiguation process in order to prevent such spurious cues from propagating into neighboring regions. In order to investigate the key computational properties of the proposed mechanism, we employ a simple X- and T-junction detector (sketched in Fig. 2, right and described in detail below) to generate a signal, which 
indicates possible occlusions. By using such a detector scheme, we simplify the general problem of detecting occlusions and assume that occlusions always generate T- or X-like form configurations.

We propose that an excitatory additive signal from the form path influences motion sensitive cells at locations of potential occlusions. At such locations with potential wrong motion information a tonic input in velocity space decreases the saliency by increasing the activity of all motion sensitive cells. This prevents those cues from propagating to neighboring locations, since the increased ambiguity generates an increased inhibition in the normalization stage, which, in turn, shunts down the entire activity pattern. This dynamic interaction is schematized in Fig. 3 showing how lateral inhibition weakens a potentially wrong motion cue. The resulting decrease of activity in the presence of occlu-

(a)

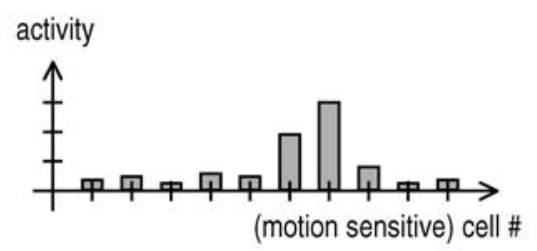

(b)

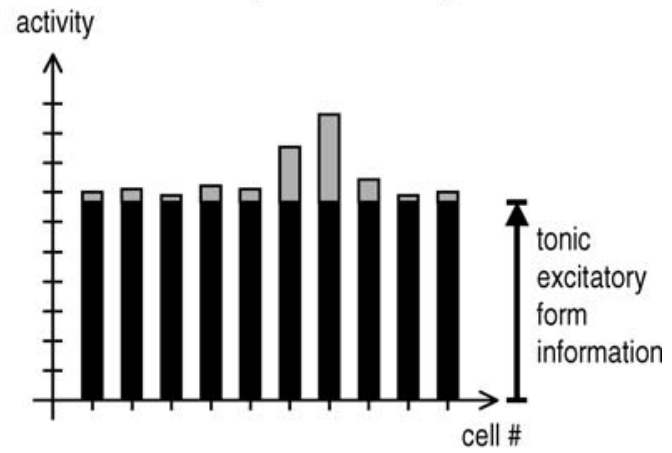

(c) activity

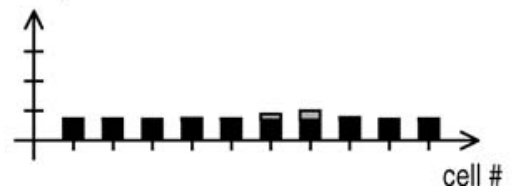

Figure 3. Schematic illustration of the influence of form information on the motion integration process, (a) shows a detected motion pattern for a subpopulation of 10 motion sensitive cells indicating a (potentially erroneous) velocity by a single peak, (b) illustrates the effect a tonic input from the form pathway, which induces an ambiguity. The additional input is generated in the form path at locations wheie possible occlusions are detected. After normalization through mutual competition (c) the maximal activity of the modified motion patterns is strongly decreased, which prevents that this potentially erroneous information is propagated to neighboring locations. sions has been reported for cells in macaque area V1 by Pack et al. (2004). The authors showed a strong suppression of direction selective V1 neurons in the presence of occlusions, independent of the orientation of the occlusion.

In order to achieve the described effect the input from the form path is added to $u^{(1)}$ after feedback modulation (see Eq. 4). Else, if it is added to the input signal net ${ }_{I N}$ before feedback modulation, motion information from different objects can be combined through propagation (such as in Fig. 1c, inlay A). If it is added after the feedforward integration, a stronger additive signal is needed, since the nonlinear signal transform (squaring) and the spatial integration may have already generated a less ambiguous motion pattern.

$$
u_{x, v}^{(1)}=n e t_{I N} \cdot\left(1+C \cdot n e t_{F B}\right)+n e t_{E X T}
$$

where net $_{\text {EXT }}$ denotes the additional external input in model V1 processing that is delivered by the form path indicating $\mathrm{X}$ - and T- junctions at each image location $x$ (index $x$ omitted). $n e t_{E X T}$ is set to zero for model area MT, since cells in model MT do not require suppressing potentially wrong cues if all such cues in model V1 were already extinguished.

\section{Generation of Localized form Signals}

The following operations are used to generate the form signal indicating T- or X-junctions. First, normalized oriented complex cell responses $c$ are computed for eight orientations $\alpha$ (Eq. 5; these complex cell responses are also utilized for the generation of the initial motion patterns in model V1 by the spatio-temporal correlation of $c$ ):

$$
c_{x, \alpha}=\frac{I^{*} \partial_{x x(\alpha)} G_{\sigma}}{0.01+\sum_{\beta}\left|I^{*} \partial_{x x(\beta)} G_{\sigma}\right|^{*} G_{\sigma}}
$$

I denotes the current frame of the image sequence (we have omitted the index $x$ for better readability), "**" the convolution operation, and $\partial_{x x(\alpha)} G_{\sigma}$ the second spatial directional derivative of a Gaussian (with $\sigma=1.0$ ) in direction $\alpha$. Second, for each orientation $\alpha$, we combine nearby complex cell responses to generate an oriented T- or X-junction measurement $\left((\mathrm{AB}(\mathrm{C}+\mathrm{D}))^{1 / 3}\right.$, see Fig. 2 and Eq. 6). We then sum these junction measurements over all orientations in a certain neighborhood (defined by a Gaussian $G_{\sigma}$ with $\sigma=2.0$ ) to yield an 
orientation independent T-junction measurement used as form input net ${ }_{E X T}$ to the motion pathway (Eq. 6):

$$
\begin{aligned}
\operatorname{net}_{E X T}(x)= & 100.0 \cdot \sum_{\alpha}\left(A _ { x , \alpha } B _ { x , \alpha } \left(C_{x, \alpha}\right.\right. \\
& \left.\left.+D_{x, \alpha}\right)\right)^{1 / 3 *} G_{\sigma}
\end{aligned}
$$

$$
\text { with } \begin{aligned}
A_{x, \alpha} & =\sum_{i=1}^{2} c_{x+i \cdot r}\left[\begin{array}{c}
\cos (\alpha) \\
\sin (\alpha)
\end{array}\right], \alpha \\
B_{x, \alpha} & =\sum_{i=1}^{2} c_{x+i \cdot r} \cdot\left[\begin{array}{c}
\cos (\alpha+180) \\
\sin (\alpha+180)
\end{array}\right], \alpha \\
C_{x, \alpha} & =\sum_{i=1}^{2} c_{x+i \cdot r \cdot[}\left[\begin{array}{c}
\cos (\alpha+90) \\
\sin (\alpha+90)
\end{array}\right], \alpha \\
D_{x, \alpha} & =\sum_{i=1}^{2} c_{x+i \cdot r}\left[\begin{array}{c}
\cos (\alpha+270) \\
\sin (\alpha+270)
\end{array}\right], \alpha
\end{aligned}
$$

and $r=6.0$ (compare Fig. 2, right). The generated form signal is illustrated for individual examples in the results section (Fig. 8 and 9).

The described X- and T-junction detector contains key elements from (Hansen and Neumann, 2004) and (Neumann and Sepp, 1999), such as a multiplicative long-range filtering and the idea that the presence of multiple orientations at one location helps classifying the image structure. In (Hansen and Neumann, 2004) the authors present a robust measurement to extract junctions in real-world images based on neural model cell responses. The underlying long-range interactions are found in area V1 of the monkey (Kapadia et al., 2000) as well as in area V2 (Heydt et al., 1984). In cat striate cortex cells were found which show an increased response to configural form properties, such as corners or crosses (Shevelev et al., 1998). The focus of our work, however, is not on the detection of occlusions, but on the integration of an occlusion signal from the form path in our model of the motion pathway and therefore relies on the much simplified detection mechanism.

It is worth mentioning that in our model form information is not used to segment the scene or to steer the region of integration of context information, but to tag and weaken potentially erroneous motion cues at occlusions. The proposed method uses no explicit suppression of specific motion signals. Instead, a roughly localized occlusion signal is added to all velocity tuned cells in the input layer of our first model area. This leads to ambiguities which are handled by the model in the same way as other ambiguities (such as the aperture problem). Thus, our approach is a novel neurally plausible realization of a SJI-model (suppress junctions and integrate) realized by a simple addition of a model ventral signal to cell activities of a previously presented model of the dorsal pathway.

\section{Results}

The results were all generated with the same parametrization of the model (see model description). The only difference is that for computational model investigations of motion integration and segregation without form interaction, the input net $_{E X T}$ is set to zero. The results shown in all figures represent or are deduced from the neural variable $u^{(3)}$.

\section{Motion Integration (without form Interaction)}

Fig. 4 demonstrates how our model processes scenes without occlusions. At line endings or corners correct and unambiguous motion estimates are indicated by local flow estimates that were detected from the beginning at stimulus onset. Along elongated contrasts, on the other hand, an initial ambiguity is present (aperture problem) that is resolved through temporal processing by propagating salient motion features from line endings or corners along object boundaries. This propagation process demonstrates that the model is able to resolve ambiguities in images with objects of arbitrary size, where larger objects are processed in the same way as smaller objects, but take longer to propagate the unambiguous information over the entire object. Thus, scale invariance of the ability to segment visual motion is achieved through temporal integration. Importantly, the relaxed model state describes image motion which contains combined motion cues from potentially any image location which were combined by purely local interactions. The result also shows that, due to the local interactions, the output of the model remains spatially segregated and image sequences with multiple objects can be processed.

Feedback vs. hierarchical filtering. Despite the fact that the model performed well in the example sketched in Fig. 4, the question arises, whether similar performance could also be achieved with a strict feedforward hierarchy of model areas with increasing receptive field sizes. This idea suggests that objects of a certain size are processed by cells with appropriate receptive field sizes. The problem which arises with such an architecture is illustrated in the following example. A temporal sequence that shows a moving 

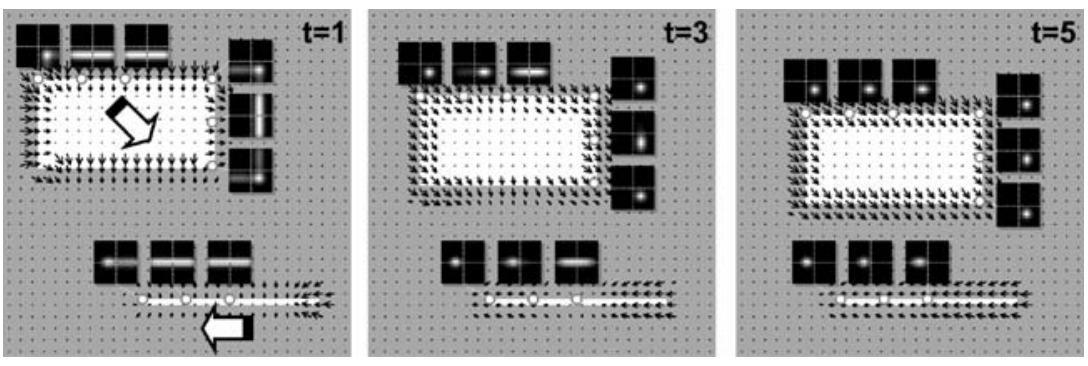

Figure 4. Results of motion segregation for an artificial test sequence without occlusions and net $_{E X T}=0(100 \times 100$ pixel $)$ after 1,3 , and 5 iterations of feedback processing. Small arrows represent velocities indicated by cell populations $u^{(3)}$ in model V1 (The normalized sum of the velocity vectors to which the cells of a population are tuned to, weighted with the corresponding activities determines the detected velocity at each location). Snapshots of the population code are plotted at individual locations as indicated by small white probes each showing the velocity space at this position (dark = low activity, light $=$ high activity). The illustrated motion patterns represent the activity distribution in the local velocity space, with its origin in zero-motion. Outlined Arrows indicate true directions of shape motion Salient features propagate from line endings and corners along extended boundaries with initially ambiguous information.

square is processed by different hypothetical model areas with different receptive field sizes build upon model V1 (we simply varied the receptive field size of cells in model area MT). The results in Fig. 5 show that for receptive field sizes which solve the aperture problem the spatial localization is completely lost, such that the precise position of a moving feature cannot be extracted from the resulting optic flow estimations. Thus, a hierarchical feedforward version of our model with simple Gaussian receptive fields and no additional mechanisms would not be able to successfully process motion sequences of objects with arbitrary size.

Concluding from Fig. 5, an important implication from feedback is that spatial accuracy is preserved by combining estimations generated in different spatial scales. In the presented model this is achieved by the interplay of two structurally identical model areas, but could this also be achieved by only one model area? To simulate this, we modified our model in the following way:

- model area V1 is almost completely omitted (raw motion estimations are directly fed to the input to model area MT, no nonlinearities and no feedback were applied) and

- feedback from the output of model MT is linked directly to the input of model MT (intra-areal feedback, see Fig. 6b).

Motion interpretations were extracted from the subpopulations of model area MT at stage $u^{(1)}$ (after the feedback modulation). The results in Fig. 6 show that with only one model area the disambiguation process is much slower. This can be explained by the fact that nonlinearities and the normalization in V1 already amplify unambiguous local motion cues, which then contribute more activity to model area MT. Our results also indicate that certain motion cues are ignored by the model if they are too small to generate a salient signal on the coarser scale of model MT.

Texture defined vs. boundary defined motion and real images. In image sequences with textured objects, occlusions typically are not as problematic as for untextured objects. Fig. 7 illustrates that the model without form information does generate correct flow estimations for overlapping textured rectangles, while without texture erroneous motion occurs. Dense textures generate coherent unambiguous correct motion cues at many locations which are assembled in model MT and reinforced in model V1. At locations with occlusions the model generates high activities for two different velocities which inhibit each other (shunting inhibition; see Fig. 7b,c). Without textures, only the object boundaries generate a motion signal. At occluding boundaries objects moving in different directions may generate a unique feature which indicates a single incorrect coherent motion over a period of time. In contrast to textured objects, boundary defined motion cues at occlusions are salient and unambiguous (see Fig $7 \mathrm{a}$ ). Thus, in typical natural sequences without regions with perfectly homogeneous luminance our model will not have great difficulties extracting good motion estimations and problems will only occur for untextured objects. 

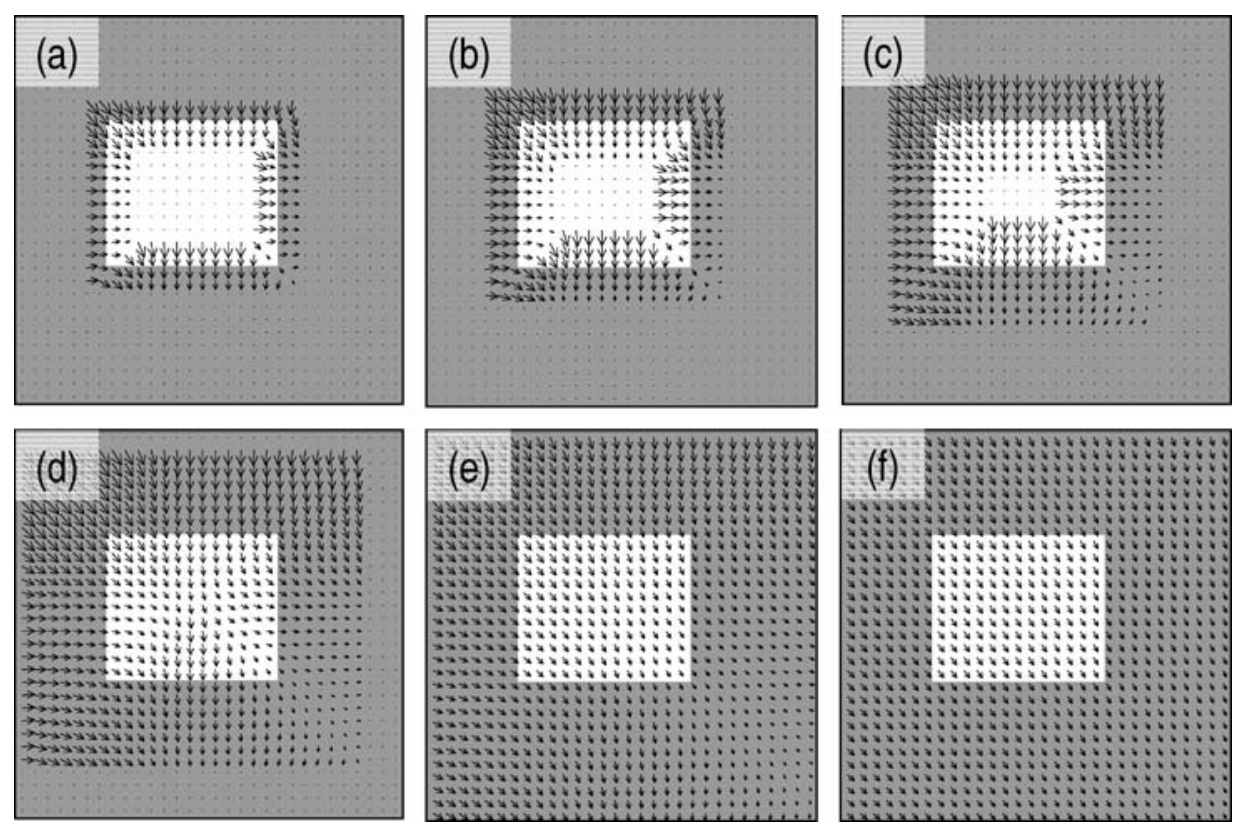

Figure 5. Results of feedforward motion estimations with increasing receptive field sizes. (a) motion estimation of model area V1 for square moving in diagonal direction to the bottom right. (b-f) motion estimations with receptive fields which double their size from one subfigure to the next. (d) corresponds to the receptive field size used in model area MT. For very large receptive fields (f), which cover large parts of the object of interest, the aperture problem is solved. However, the spatial localization is completely lost, such that the precise position of a moving feature cannot be extracted from the resulting optic flow estimations.

Importantly, the described form-motion interaction does not harm the motion segmentation process in the presence of textures. Although the form interactions seems unnecessary for image sequences with dense textures it supports the segmentation of the scene by further decreasing the maximal neural activity at occlusions and thus represents a unifying mechanism to deal with motion signals in the presence of occlusions.

Fig. 8 presents results of processing a real-world example showing a tree moving differently than the background caused by the motion parallax for an observer moving to the left. Motion is segregated without any additional form information and the boundary of the tree is indicated by low values of maximal activity at the corresponding locations. Thus, no unambiguous erroneous motion cue was generated due to occlusions in this example. The background of the scene contains objects at different depths (the flowers and the houses) generating a velocity gradient which is successfully detected and represented by the model. This velocity gradient deviates from the assumption of coherent motion. The smoothing term in Eq. 2 operating in velocity space allows similar velocities to be pooled to- gether in both model areas and thus allows gradual changes of velocity to be integrated and represented by the model.

\section{Form-Motion Interaction}

In the presence of overlapping objects or transparency, detected motion cues near occlusions often indicate erroneous motion (Fig.1c). Here, we show that the proposed form-motion interaction can help to disambiguate sequences even in the presence of such error prone input patterns.

In Fig. 1 and 7 we demonstrate which problems arise in the presence of overlapping objects and how the proposed form-motion interaction contributes to the solution of the problem. Figure 9a shows how occlusions can lead to erroneous initial motion estimates in a sequence where two overlapping diamonds move in opposite directions (McDermott et al., 2001). In the spatial neighborhood of occlusions unambiguous motion signals arise which, however, indicate wrong movement directions. These erroneous motion cues 
(a)
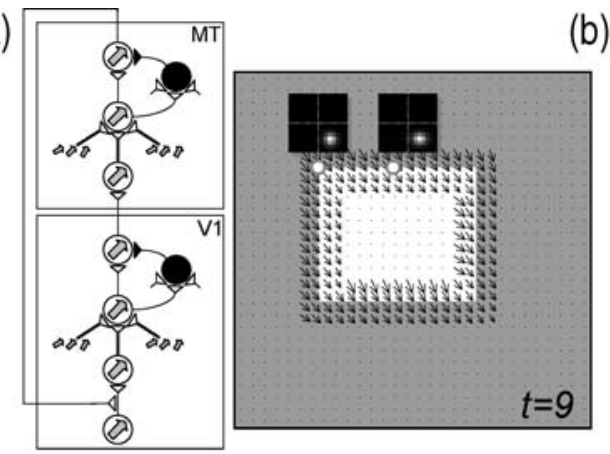

(b)

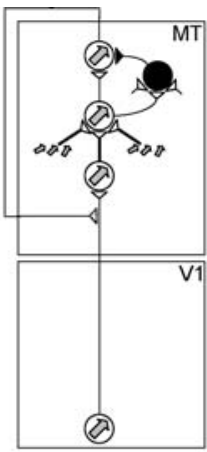

(c)
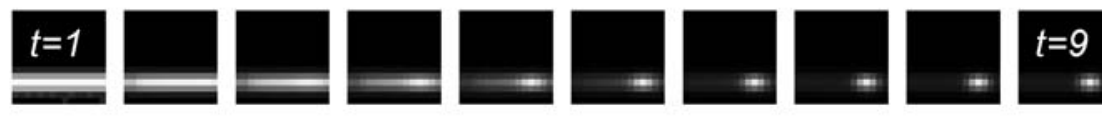

(d)
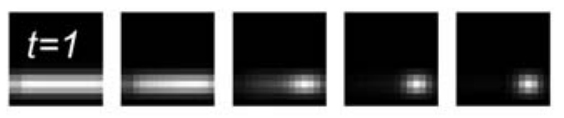

(e)

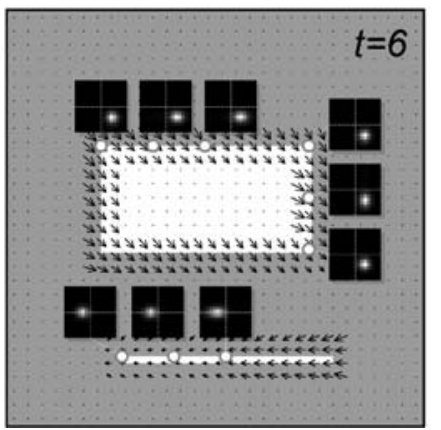

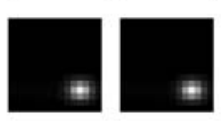
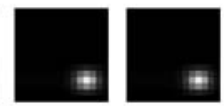

(f)

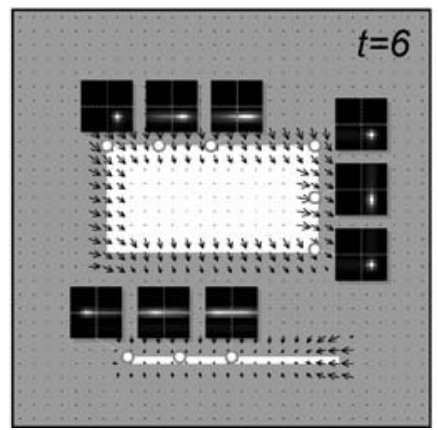

Figure 6. Experiment illustrating the role of two areas operating on different scales. (a) sketch of the presented (full) model (see section "Methods" and Fig. 2) and estimated velocities after 9 iterations of feedback processing of a square moving diagonally to the bottom right. (b) shows a model variation and estimated velocities where information processing in model area V1 is omitted and feedback directly modulates the input to model area MT (intra-areal feedback). This model variation has the disadvantage that small unambiguities in model V1 are not stressed by local competition and may be blurred away by the spatial integration in model MT. (c,d) show the population code in the center of the top edge of the square at different time steps (measured in iterations) of both model variations (a) and (b), respectively. (d) Without additional signal processing in model area V1 the disambiguation process takes much longer and is still influenced by form constraints (aperture effect) after 9 iterations. (e) and (f) illustrate the result of motion estimation of the sequence described in Fig. 4 after 6 iterations of feedback processing. Without the additional amplification of unambiguous signals in model V1 (f), the propagation is much slower. Especially very small features (e.g. as the left line ending) are not dominant enough to even initiate the disambiguation process.

propagate into neighboring locations, which leads to regions that indicate completely wrong motion signals. Figure $9 \mathrm{~b}$ demonstrates how additional form information indicating possible occlusions influences the model dynamics. The occlusion signal creates an ambiguity through direct excitation of all velocity dependent cells in V1 at the corresponding location. Subsequent lateral shunting competition (divisive inhibition) between responses leads to an activity normalization and, in turn, to a net inhibition of such erroneous signals. As a result, no salient features emerge that point to erroneously improper directions, which then could be propagated and enhanced by feedback interactions. Conversely, salient features from other corners of a unique surface patch fill-in regions along the boundaries with unambiguous motion signals.

Similar effects as for overlapping opaque objects (Fig. 1 and 9) can occur in the presence of transparency. Figure 10 illustrates such an example where two overlapping moving bars are viewed through a rectangular aperture in which the vertical bar is moving horizontally, whereas the horizontal bar is moving vertically. In the non-transparent case (Fig. 10a) the opaque bars are perceived as one diagonally 

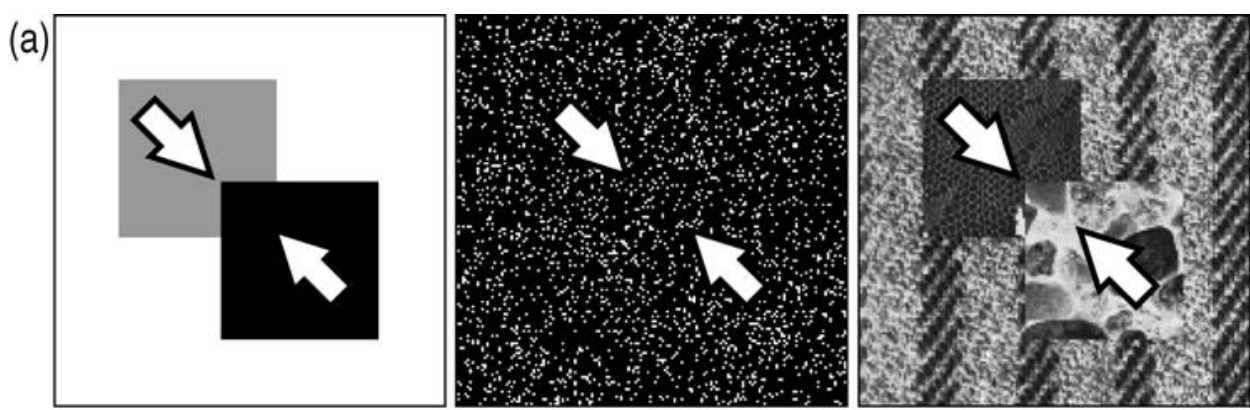

(b)
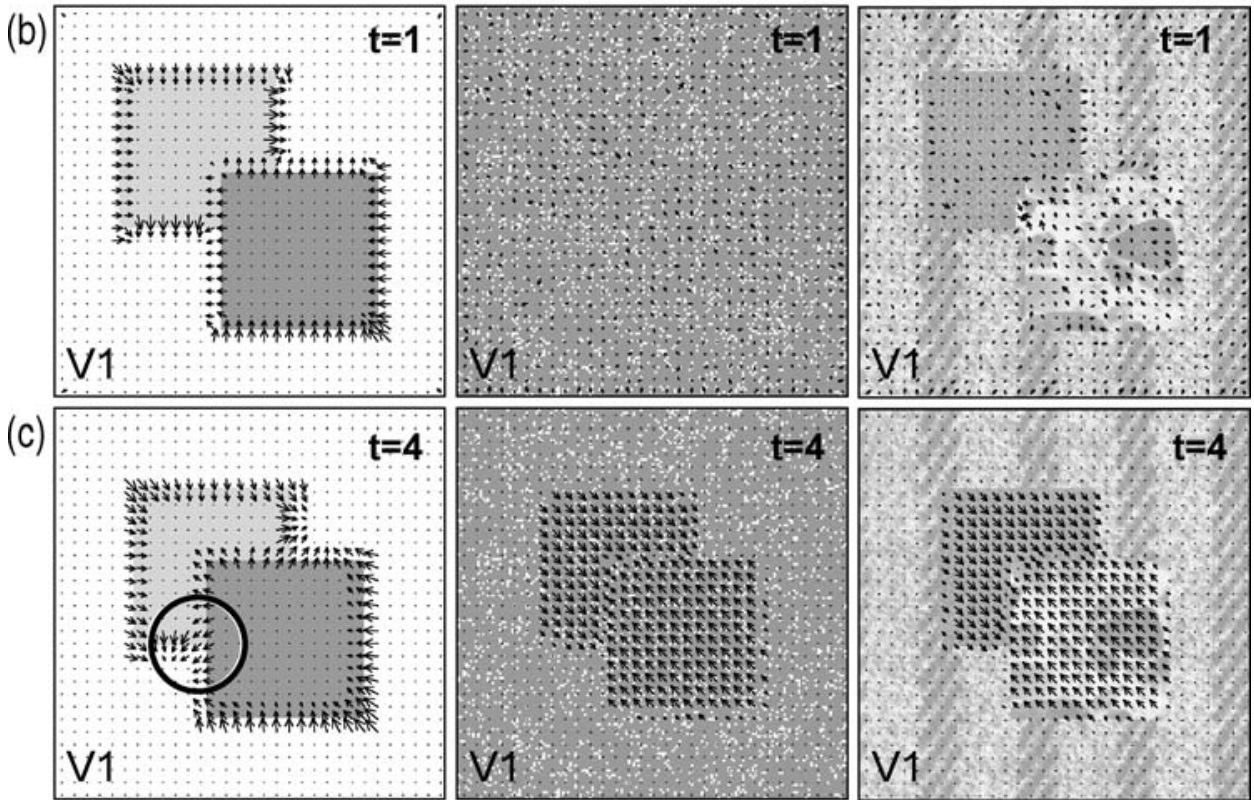

(d)
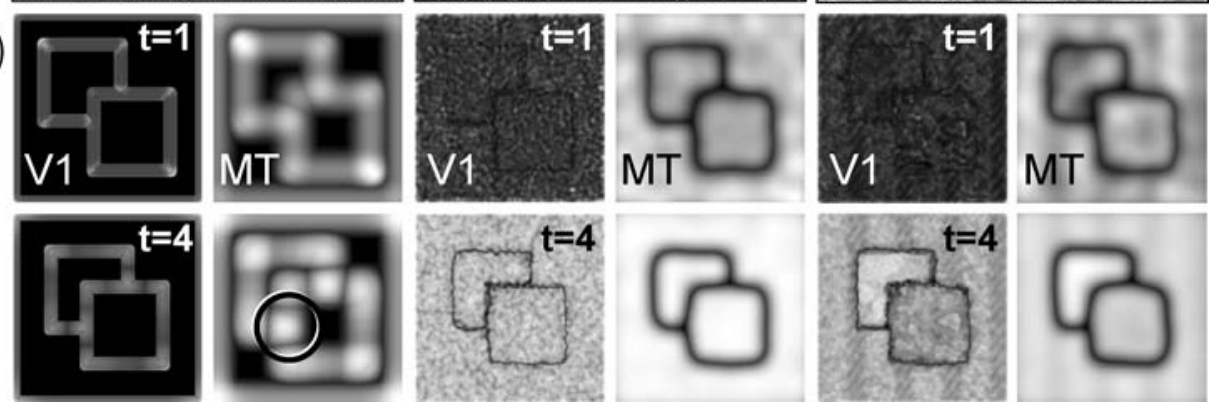

Figure 7. Comparison of results with boundary-defined and texture-defined motion in the presence of occlusions. (a) First frame of the employed image sequences. Two overlapping squares moving in diagonal direction over a static background plane of homogeneous luminance (left), textured with random dots (center), or with Brodatz textures (right). Motion is indicated by outlined arrows. (b) initially detected motion and (c) motion indicated after 4 steps of feedback iteration (shown: velocities indicated by cells $u_{3}$ in model V1). In the sequence without texture (left) erroneous motion is estimated at occlusions with the other object. For boundary-defined motion erroneous unimodal motion patterns indicating a single velocity stabilize and propagate their information. This can be seen by an increased neural activity at occlusions indicating salient motion, e.g., at the marked location in the output of model area MT (d, left). In (d) luminance encodes $\max \left(u_{3}\right)$ taken over all velocities at each location at different time steps. In both textured sequences (center, right) the model without form information generates dense optic flow estimations (c). Correct motion cues are indicated within the object, while motion at occlusions (with ths other object and the backgound) are suppressed. This is explained by the fact that inhomogenities of the surfaces typically generate two motion cues near occlusions, pointing in the directions of both object movements instead of generating consistent patches of a single wrong velocity. In textured sequences such bimodal motion patterns are inhibited by the shunting inhibition (Eq. 2) and thus may not propagate their information into neighboring region. The reduced neural activity can be observed near occlusions ( $\mathrm{d}$, center and right), which outlines the objects of the scene. Consequently, wrong motion cues from occlusions play a minor role for densely textured objects. 
(a)
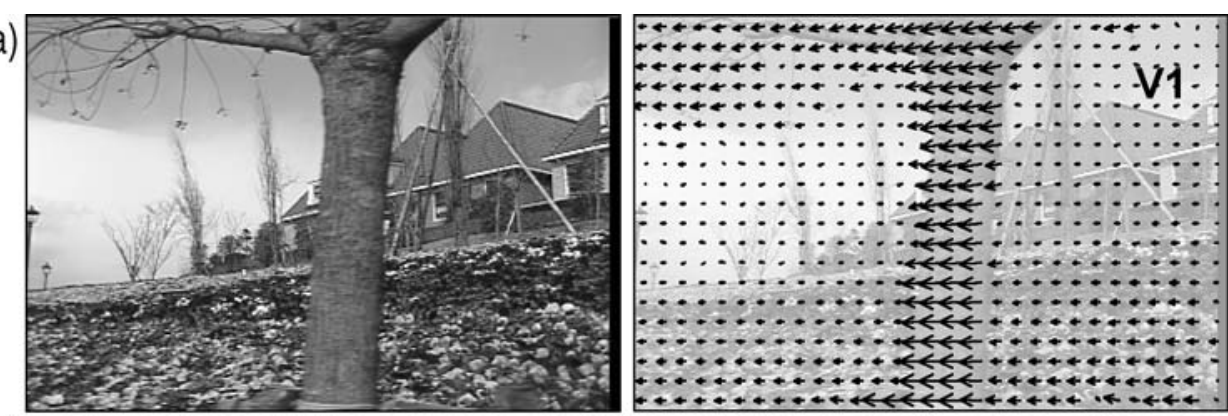

(b)
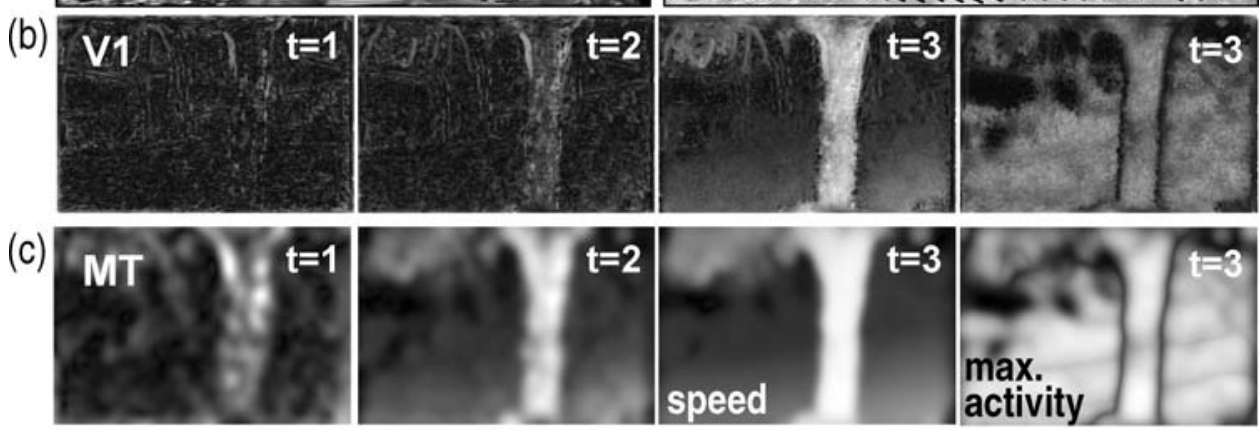

(d)
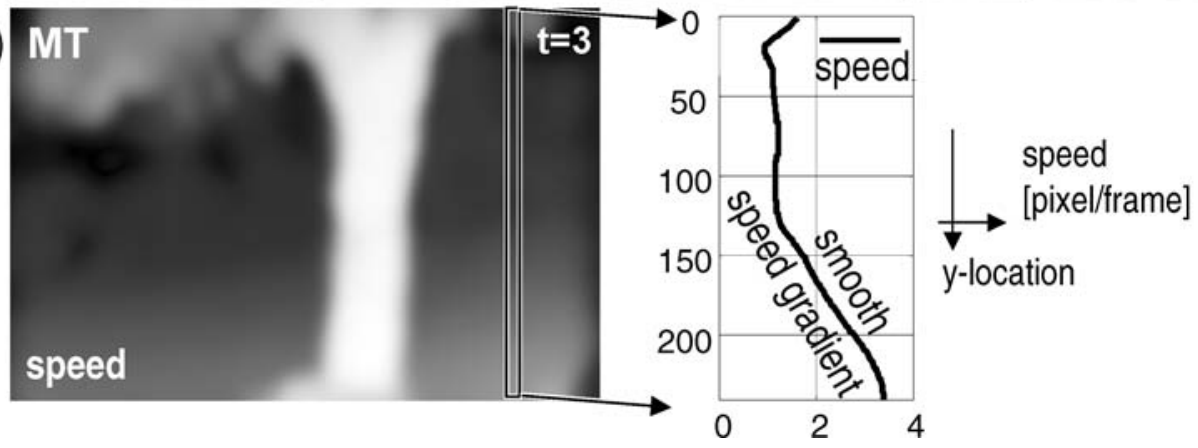

Figure 8. Model results processing a real-world sequence including occlusions and a velocity gradient. The input sequence shows a tree moving differently as the background due to a movement of the observer to the right (http://www.cs.brown.edu/people/black/images.html). (a) the 7th frame of the sequence and the motion indicated by the model (population $u_{3}$ of model V1; the normalized sum of the velocity vectors to which the cells of a population are tuned to, weighted with the corresponding activities determines the detected velocity at each location). (b,c) shows the velocities indicated in both model areas at different time steps $\left(t=1,2,3\right.$, left images) as luminance and $\max \left(u_{3}\right)$ taken over all velocities at each location (for $t=3$, right image). This example shows that in typical natural scenes occlusions mostly induce texture-defined motion discontinuities which lead to low activities around objects. (d) illustrates the ability of the model to detect and represent speed gradients. For the marked column in the image the detected speed is shown (right). The speed gradient induced by the flowers arranged at different depth is clearly visible. Locations farer away from the observer lead to lower speeds than structures near to the observer.

moving cross, while in the transparent case (Fig. 10b) the transparent bars are perceptually split into separate objects moving in either horizontal or vertical directions, respectively. A similar stimulus was presented by Stoner et al. (1990) with overlaid gratings. Consistent with perceptual observations, the computational model estimations indicate one direction in the non-transparent case and multiple directions for the transparent case, pointing roughly in vertical or horizontal directions. In order to switch be- tween the two motion interpretations, Stoner at al. (1990) suggest that the visual system employs some "tacit 'knowledge' of the physics of transparency" (p. 153). Our investigations show that such a behavior can be achieved by the dynamics of our model based on local form configurations that also occur for nontransparent occlusions (as in Fig. 9). However, we do not rule out that some additional information, such as the direction of figure (DOF), may be incorporated in this disambiguation so that it improves the processing 

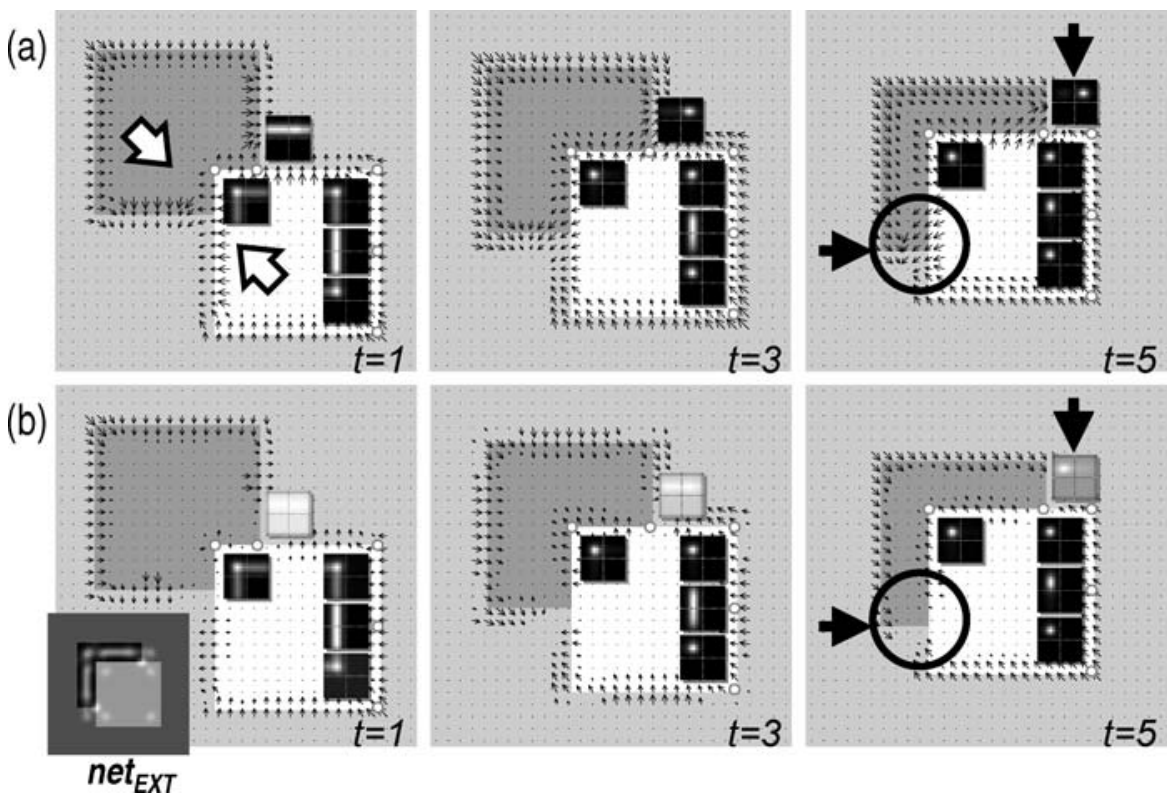

Figure 9. Results of motion segregation for an artificial test sequence with occlusions. (a) with net $_{E X T}=0$ salient junction features emerge near occlusions that represent wrong motion signals pointing orthogonal to the true direction of motion (locations indicated by bold arrows for $t=5$ ) (b) with net $_{E X T} \neq 0$ indicating possible occlusions (see inlay: net $_{E X T}$ is overlaid over the input image) only correct features propagate and thus, the correct shape motions are finally represented (indicated by bold arrows for $t=5$ ). Note, that no arrows illustrate the correctly detected direction indicated by the population code in (b), $t=5$, which still contains a not negligible amount of ambiguity. This caused by the vector sum utilized to interpret the population code (compare Fig. 4).

of more complex figures. In the presented example we assume the $\mathrm{X}$-junctions in the scene indicate occlusions. In order to correctly account for transparency effects, it would be important to also include information about luminance ratios between surfaces (Metelli, 1974) to generate a correct local form signal indicating transparency and thus occlusions by overlapping surfaces. In order to keep the model simple we did not include such information.

\section{Discussion}

In this section we discuss the model and the presented results in comparison to other computational approaches. First, we compare the mechanisms of motion integration and segregation (without form interactions) to other models. Then we discuss the proposed influence of form information on mechanisms of the motion pathway.

The motion integration and segregation process is realized by bidirectional interactions between two model areas. Mechanisms of feedforward integration (motion detection and spatial integration) and lateral interac- tions (normalization) utilize similar mechanisms as in other approaches (Adelson and Bergen, 1985; Weber and Malik, 1995; Simoncelli and Heeger, 1998). Unlike those approaches, our model does not generate velocity estimations directly on the basis of such feedforward activity patterns. Our simulations demonstrate that the feedforward version of the model cannot resolve ambiguities caused by the aperture problem and at the same time preserve the spatial localization. Thus, a new contribution of our model is the recurrent spatiotemporal combination of information by feedback modulation, which is used to decide which velocities at each location are relevant for the global interpretation of visual motion. The mechanism can be interpreted as a neural implementation of the IOC approach (Adelson and Movshon, 1982). The interplay of the described mechanisms in an iterative loop realizes a spreading of disambiguated information into regions with ambiguous motion estimations.

Our model simulations demonstrate that the presented model with two bidirectionally connected model areas leads to better results compared to a model with only one model area and intra-areal feedback, or long-range, connections: the disambiguation process 

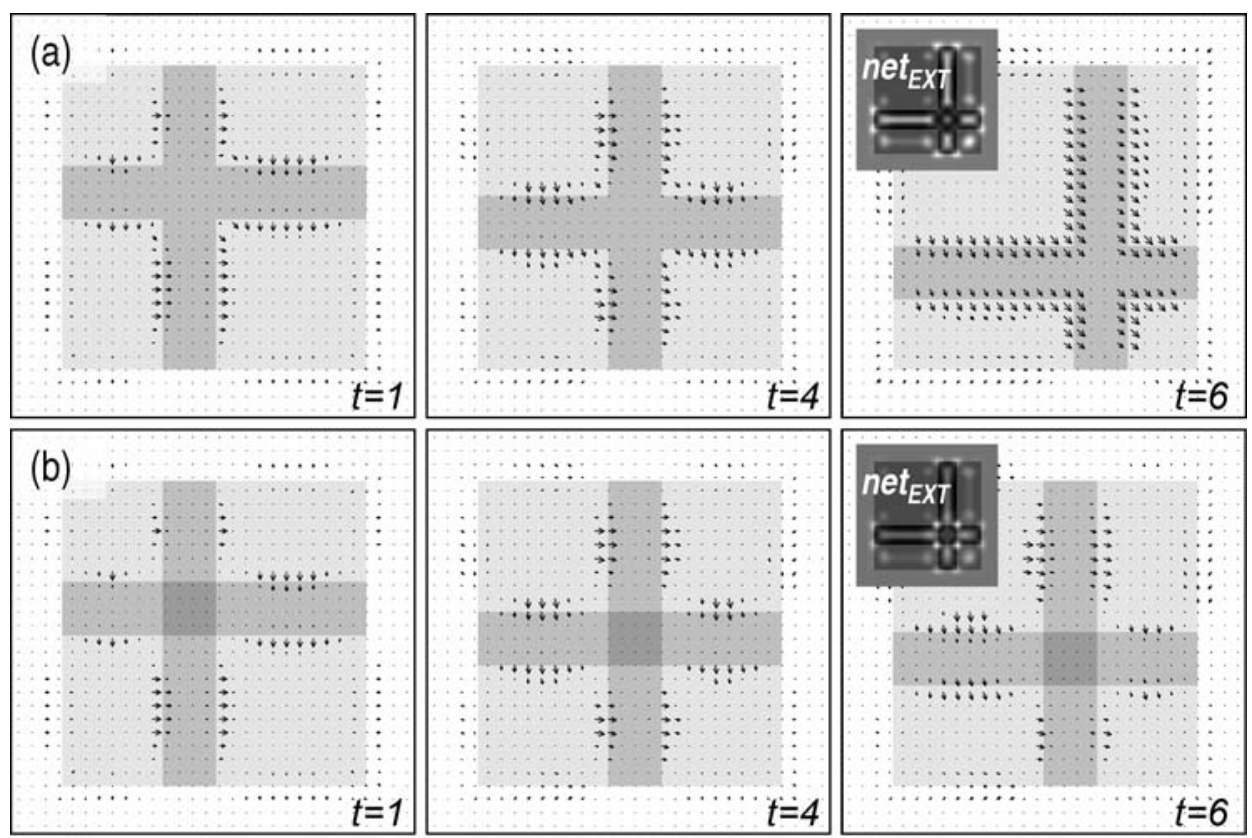

(c)

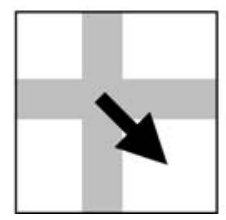

(d)

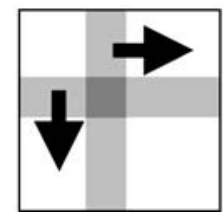

Figure 10. Results of motion segregation for two artificial test sequence of opaque and transparent bars moving through a rectangular aperture. Without transparency (a) the sequence is perceived as one object moving in right downward direction, compare (c). In the presence of transparency

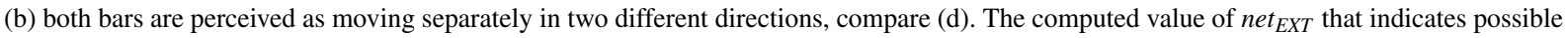
occlusions is 0 near the center of the cross for opaque bars (a, see inlay), while in the presence of transparency (b) net $t_{E X T} \neq 0$ at occluding junctions (see inlay). Consistent with perceptual observations the motion signal is separated in (b) indicating roughly horizontal and vertical motion, while one single object moving in right downward direction is detected in (a).

is much faster and sharper in velocity space with two model areas and, in some situations, the motion disambiguation with only one model area fails to extract the correct solution. Such a single area implementation of our model is comparable, but not identical to models with recurrent lateral interactions, where information from neighboring locations is combined in a recurrent loop. The approach of, e.g., Koechlin et al. (1999) is a model with such recurrent lateral connections. The authors utilize a gating process to combine neighboring motion estimations in a Bayesian-like manner. Unlike the soft-gating employed by our model, such an interaction includes excitatory as well as inhibitory feedback information, which may lead (numerically) to a complete inhibition of relevant information in the input signal and, thus, lead to wrong motion estimations. Furthermore, the results published in Koechlin et al. (1999) show that they fail to solve the aperture problem for moving bars in cases when they are longer than the size of their receptive fields. Lidén and Pack (1999) proposed another model of recurrent lateral motion interactions, which is able to produce a traveling wave of motion activation to solve the aperture problem. Like our model, they use a normalization mechanism similar to the one described by Simoncelli and Heeger (1998) to emphasize salient motion estimates. In contrast to our model, their normalization mechanism is not isotropic in the velocity space and the proposed mechanisms lead to an unbounded filling-in process which has to be constrained by long-range inhibition of motion cells of different directional selectivity and by a motion boundary signal. Such motion boundaries were generated by an auxiliary process to support the motion disambiguation.

In technical terms, our approach represents a voting scheme like the Hough transform (Hough, 1959). 
Our model explicitly represents cells for individual solutions of the motion correspondence problem. For a given input the cell with the maximum response indicates the most likely solution. At a given time, the input to a cell is defined by the bottom-up input (feedforward motion detection and spatial pooling) and a recurrent top-down signal which together define the spatiotemporal dynamics of the system. The computation of the initial cell activities in model V1 (corresponding to initial likelihoods of individual velocities) is realized by evaluating the similarity of the corresponding regions of the input frames. The underlying assumption for the initial motion estimation is that local orientation configurations on the spatial scale defined by the simulated complex cell responses (see Method section) remain constant within objects over time and the computed similarity measure is similar to the normalized cross-correlation (compare Bayerl \& Neumann, 2004). Similar initial motion signals (or motion hypotheses) can be computed based on, e.g., gradient based methods, phase based methods, or by comparing wrapped image regions (for an overview see Barron et al., 1994). Despite the coarse sampling of the velocity space by cells tuned to integer velocities our model is able to interpolate between these velocities (as shown in Fig. 8).

In terms of Marr's computational approach to vision (Marr, 1982) (1) the computational theory is to detect motion correspondences in a sequence of frames based on bottom-up similarities from the input and top-down expectations from previous observations. (2) The representation and the algorithm are given by the model representation as population code and the composition of neural mechanisms, which in concert solve the given problem. (3) The hardware implementation is not provided, but the simplicity of the employed mechanisms and the ability to perform most of the operations in parallel suggests an efficient hardware realization.

The interpretation of neural activity as likelihoods is comparable to Bayesian models (Weiss et al., 2002; Lee and Mumford, 2003). Our feedback signal can thus be interpreted as dynamic prior for the actual observation. In contrast to Bayesian models, our "prior" changes over time and depends on previous estimations in a small neighbourhood. Also, activities (likelihoods) of nearby estimations which are pooled together cannot be assumed to be the outcome of statistically independent processes. Moreover, there exist alternative voting schemes such as tensor voting (Medioni et al., 2000). In the tensor voting approach the data representation is highly compressed and encodes additional information about local data distributions in $\mathrm{nD}$ space $(4 \mathrm{D}$ for motion; Nicolescu and Medioni, 2003). In contrast to tensor voting, the population approach employed in our model represents individual characteristics of the represented data (velocities) without having additional information about actual data distributions that are implicitely represented in the signal.

Our model has to be distinguished from approaches where information about local luminance contrast orientation (Nicolescu and Medioni, 2003; Weiss and Adelson, 1996) or motion discontinuities (Nagel and Enkelmann, 1986; Brox et al., 2004) is utilized to steer the direction of integration of motion information. We use local form configurations to inhibit possibly erroneous motion cues, and employ a motion integration scheme based on spatial kernels of fixed size, comparable the window function of Lukas and Kanade (1981) on one single spatial scale. Our model has also to be distinguished from other approaches which try to separate multiple motion signals at one given location in the case of transparency or occlusions (e.g., Nestares and Fleet, 2001). The presented model utilizes small receptive fields and we assume that the majority of RFs typically do not see more than one object motion. If receptive fields cover regions with object boundaries then they detect multiple motion cues and thus generate very weak motion responses caused by the lateral inhibition (normalization). Importantly, the model allows that motion belonging to one object may contain small motion gradients, which is realized by the smoothing kernel applied in velocity space (compare Eq. 2 and Fig. 8). Furthermore, the sequence includes a velocity gradient which is detected by the model. The new contribution in this paper is that we propose a framework to segment motion signals belonging to different objects which handles erroneous motion cues induced by occlusions. Such erroneous local flow estimations typically occur over a longer time period in a consistent configuration at occlusion of non-textured objects (boundary defined motion; compare Fig. 7 and 8). To extract candidates for this type of occlusions we apply a simplified occlusion detector and feed the extracted occlusion signal into the motion pathway as described in Model section.

Grossberg et al. (2001) studied how motion signals from partly occluded patterns can be integrated and segregated in a recurrent fashion including feedback between two different model areas. In contrast to our approach, their feedback signal (from MST) inhibits 
MT activities and is (more) global due to the receptive field size of model MST cells (depending on the stimulus, these receptive fields cover $50 \%$ up to $100 \%$ of the entire stimulus). As a consequence, the resulting flow fields are not localized spatially. Unlike our model these authors do not demonstrate to produce spatially segregated motion estimations and they do not show how information may be propagated beyond the range of their largest receptive fields (in MST). Thus, we claim that their model falls short of processing objects of arbitrary size. Our model, on the other hand, realizes such size invariance through the propagation of salient features and at the same time preserves the fine localization of initial motion estimations. Furthermore, a property which seems unique to our model compared to other models of motion integration and segregations is that it consists of two model areas based on a structural identical architecture which only differ in their receptive field sizes which achieve combining context information with a spatially localized input signal.

Weiss and Adelson (1994) presented a model which uses global motion information combined with local interactions from the form path, whereas our model only employs local interactions. In the presence of potentially erroneous input cues, such as for stimuli similar to the moving diamonds (Fig. 1c) the authors apply a segmentation of the image in the form pathway in order to correctly estimate object motion. This differs from our model, where no complex structural information, such as a segmented image, needs to be delivered from the form path. Furthermore, our model segments the image based on motion information influenced by local form cues, instead of refining a global segmentation from the form path by using motion information. However, our model only estimates motion in the presence of some gray level variation (e.g. along object boundaries), whereas Weiss and Adelson (1994) are able to fill-in regions of homogeneous luminance. We appreciate that information about the DOF, which is used by (Sajda and Baek, 2004) and (Weiss and Adelson, 1994) play a major role in the interpretation of motion of moving forms and may be the key feature to trigger a filling-in process of motion estimations into unstructured regions with an intrinsic dimensionality of zero (i0D). However, we were interested in developing mechanisms of local form-motion interaction to demonstrate the segmentation capabilities of motion segmentation on a neural basis. The integration of global mechanisms in our model in addition to local interactions is beyond the scope of this paper.
Local form information utilized by our model is generated by a very simple operation to determine T- or Xjunctions (see Model section). As a consequence, the form signal utilized in the presented contribution accounts for occlusions only if they are represented by Tand $\mathrm{X}$-junctions and also correctly handles those junctions in case they actually represent occlusions. Other form configurations require a more elaborated model of the ventral pathway to detect occlusions. To correctly handle transparency a representation of luminance ratios between different surfaces is required (Metelli, 1974). More complex form configurations may contain nonlocal form constraints (McDermott et al., 2001) to clarify the 3D arrangement or depth order of the underlying scene elements. The DOF may be utilized to extract and propagate the desired form information along contours (Baek and Sajda, 2003). The integration of local occlusion information in the motion processing stream realized in our model basically implements the idea to suppress (or to prevent the generation of) potentially erroneous motion cues and to use the remaining information to generate globally consistent visual motion estimations. This general idea of suppressing the generation of erroneous motion cues has also been discussed in other approaches, such as (Grossberg et al, 2001; Sajda and Baek, 2004). In comparison to these approaches, the mechanism to suppress spurious motion in our model differs from all other approaches discussed above. Instead of a selective suppression of individual cells or other specialized interactions in the motion stream, we employ a mechanism that increases local activities in the velocity domain through tonic excitation. Such a simple simultaneous excitation saturates the activities of velocity tuned cells such they quickly loose their selectivity and become ambiguous. Mutual competition between those cells normalizes activities so that responses will be suppressed at locations that signal potentially erroneous motion directions. Unlike other approaches our model is able to generate localized object motion information and does not rely on global operations to integrate local form and motion patterns.

\section{Conclusion}

We presented a model of motion processing in cortical area V1 and MT of the motion path with interactions from the form path. The contributions are two-fold: First, we present a motion integration and segregation 
scheme which is able to resolve ambiguities in a scaleinvariant manner with local interactions in image sequences without occlusions. Second, we introduce a mechanism which utilizes local form information to influence the mechanisms of the motion pathway in order to correctly process motion in the presence of occlusions.

Our model is able to integrate and segregate visual motion in image sequences in a scale invariant manner. The relaxed model state represents globally consistent information, where inputs from the entire scene can potentially interact with each other. This property is achieved by local interactions in a network of recurrent feedback information processing in which feedback signals deliver a much broader scenic context to disambiguate local measures. We have shown that recurrent modulation from model MT to model V1 generates localized and segregated motion estimations, which is not possible with pure feedforward interactions. We further demonstrated that the use of two bidirectional connected model areas leads to faster and more stable results than a corresponding single-area model.

The presented form-motion interaction demonstrates how a simple mechanism of excitatory interaction may resolve potential errors of candidate motion features at extrinsic surface boundaries. It demonstrates how a combination of an input unspecific to velocity with competitive/cooperative mechanisms may suppress undesired activity patterns, while ambiguities in the remaining motion patterns are resolved in recurrent fashion. The model predicts that if the neural mechanisms of form interpretation are suppressed or the connections between form related and motion related areas are deactivated, observers will be confused by boundary defined motion in the presence of occlusions while texture defined motion is not affected.

Our model can be interpreted as a biologically plausible realization of the intersection-of-constraints (IOC) motion integration scheme, which allows to dynamically tag those constraint lines that do not belong to one single object and, in turn, to suppress their intersection vote. In other words, it realizes a segmentation of image motion on a neural basis combining local motion information from the dorsal pathway by means of the IOC influenced by local form information from the ventral pathway.

\section{Notes}

1. The oriented complex cell responses are obtained by filtering the input images twice with oriented first derivatives of Gaussian fil- ters $(\sigma=0.75)$, normalized over the entire range of 8 orientations by divisive inhibition (compare Eq. 5 in subsection "Form-motion interaction").

2. Similar results for getting initial motion estimations can be obtained by correlating Laplacian filtered input images or by directly correlating the gray level information of the input signal.

\section{References}

Adelson, E.H. and Movshon, J. 1982. Phenomenal coherence of moving visual patterns. Nature, 300:523-525.

Adelson, E.H. and Bergen, J. 1985. Spatiotemporal energy models for the perception of motion. Optical Society of America, A 2(2):284299.

Baek, K. and Sajda, P. 2003. A probabilistic network model for integrating visual cues and inferring intermediate-level representations. IEEE Workshop on Statistical and Computational Theories of Vision (SCTV'03). Online document: http://www.stat.ucla.edu/ yuille/meetings/2003_workshop.php

Barron, J.L., Fleet, D.J. and Beauchemin, S.S. 1994. Performance of optical flow techniques. Int. J. Computer Vision, 12(1):43-77.

Bayerl, P. and Neumann, H. 2004) Disambiguating Visual Motion through Contextual Feedback Modulation. Neural Computation, 16(10):2041-2066.

Brox, T., Bruhn, A., Papenberg, N. and Weickert, J. 2004. High accuracy optical flow estimation based on a theory for warping. In Pajdla, T., Matas, J., editors, Proceedings of the 8th European Conference on Computer Vision, Prague, Czech Republic.

Crick, F. and Koch, C. 1998. Constraints on cortical and thalamic projections: The no-strong-loop hypothesis. Nature, 391:245-250.

Dempster, A.P., Laird, N.M. and Rubin, D.B. 1977. Maximum likelihood from incomplete data via the EM algorithm. J. R. Statist. Soc. B, 39:1-38.

Desimone, R. and Duncan, J. 1995. Neural mechanisms of selective visual attention. Annual Review of Neuroscience, 18:193-222.

Grossberg, S. 1980. How does a brain build a cognitive code? Psychological Review, 87:1-51.

Grossberg, S., Mingolla, E. and Viswanathan, L. 2001. Neural dynamics of motion integration and segmentation within and across apertures. Vision Research, 41:2521-2553.

Hansen, T. and Neumann, H. 2004. Neural mechanisms for the robust detection of junctions. Neural Computation, 16(5):1013-1037.

Heydt R., von der, Peterhans E. and Baumgartner G. 1984. Illusory contours and cortical neuron responses. Science 224: 1260-1262.

Hough, P.V.C. 1959. Machine Analysis of Bubble Chamber Pictures. International Conference on High Energy Accelerators and Instrumentation, CERN.

Hupé, J.M., James, A.C., Girard, P., Lomber, S.G., Payne, B.R. and Bullier, J. 2001. Feedback connections act on the early part of the responses in monkey visual cortex. J. of Neurophys, 85:134-145.

Kalkan, S., Calow, D., Felsberg, M., Worgotter, F., Lappe, M. and Kruger, N. 2004. Optic Flow Statistics and Intrinsic Dimensionality. Proc. 'Brain Inspired Cognitive Systems 2004' (Stirling, Scotland).

Kapadia, M.K., Westheimer, G. and Gilbert, C.D. 2000. Spatial distribution of contextual interactions in primary visual cortex and in visual perception. J. Neurophysiol, 84(4):2048-2062.

Koechlin, E., Anton, J.L. and Burnod,Y. 1999. Bayesian inference 
in populations of cortical neurons: A model of motion integration and segregation in area MT. Biological Cybernetics, 80:25-44.

Lee, T.S. and Mumford, D. 2003. Hierarchical Bayesian inference in the visual cortex. Journal of Optical Society of America, A. 20(7):1434-1448.

Lidén, L. and Pack, C.C. 1999. The role of terminators and occlusion in motion integration and segmentation: A neural solution. Vision Research, 39:3301-3320.

Lukas, B.D. and Kanade, T. 1981. An iterative image registration technique with an application to stereo vision. In Image Understanding Workshop.

Marr, D. 1982. Vision. H. Freeman and Co.

McDermott, J., Weiss, Y. and Adelson, E.H. 2001. Beyond junctions: Nonlocal form contraints on motion interpretation. Perception, 30:905-923.

Medioni, G., Lee, M.S. and Tang, C.K. 2000. A Computational Framework for Segmentation and Grouping. Elsevier Science.

Metelli, F. 1974. The Perception of Transparency, Scientific American, 230:90-98.

Nagel, H. and Enkelmann, W. 1986. An investigation of smoothness constraint for the estimation of displacement vector fields from image sequences. IEEE Transactions on Pattern Analysis and Machine Intelligence, 8:565-593.

Neumann, H. and Sepp, W. 1999. Recurrent V1-V2 interaction in early visual boundary processing. Biological Cybernetics, 81:425444.

Nestares, O. and Fleet, D.J. 2001. Detection and tracking of motion boundaries. IEEE Conference on Computer Vision and Pattern Recognition, 2:358-365.

Nicolescu, M. and Medioni, G. 2003. Motion segmentation with accurate boundaries - a tensor voting approach. Proceedings IEEE Conference on Computer Vision and Pattern Recognition, 1:382389.

Pack, C.C., Gartland, A.J. and Born, R.T. 2004. Integration of contour and terminator signals in visual area MT of alert macaque. Journal of Neuroscience, 24:3268-3280.

Sajda, P. and Baek, K. 2004. Integration of form and motion within a generative model of visual cortex. Neural Networks: Special Issue on Vision and Brain, 17:809-821.
Shevelev, I.A., Lazareva, N.A., Sharaev, G.A., Novikova, R.V. and Tikhomirov, A. S. 1998. Selective and invariant sensitivity to crosses and corners in cat striate neurons, Neuroscience, 84(3):713-721.

Shimojo, S., Silverman, G. and Nakayama, K. 1989. Occlusion and the solution to the aperture problem for motion. Vision Research, 29:619-626.

Simoncelli, E.P. and Heeger, D.J. 1998. A model of neuronal responses in visual area MT. Vision Research, 38:743-761.

Sporns, O., Gally, J.A., Reeke, G.N. and Edelman, G.M. 1989. Reentrant Signaling among Simulated Neuronal Groups Leads to Coherency in their Oscillatory Activity. PNAS 86: 7265-7269.

Stoner, G.R., Albright, T.D. and Ramachandran, V.S. 1990. Transparency and coherence in human motion perception. Nature, 344:153-155.

Van Essen, D.C. and Galant, J.L. 1994. Neural mechanisms of form and motion processing in the primate visual system. Neuron, 13:110.

Weber, J. and Malik, J. 1995. Robust computation of optical flow in a multi-scale differential framework. Int. J. Computer Vision, 14:67-81.

Weiss, Y. 1997. Motion Segmentation using EM-a short tutorial. Online-document: http://www-bcs.mit.edu:16080/people/ yweiss/emTutorial.pdf

Weiss, Y. and Adelson, E.H. 1994. Perceptually organized EM: A framework for motion segmentation that combines information about form and motion. MIT Media Lab Perceptual Computing Section TR \#315.

Weiss Y. and Adelson E.H. 1996. A unified mixture framework for motion segmentation: incorporating spatial coherence and estimating the number of models. Proceedings of IEEE conference on Computer Vision and Pattern Recognition. 321-326.

Weiss Y., Simoncelli E.P. and Adelson E.H. 2002. Motion Illusions as Optimal Percepts. Nature Neuroscience, 5(6): 598-604.

Zetzsche, C. and Barth, E. 1990. Fundamental limits of linear filters in the visual processing of two dimensional signals.Vision Research, 30(7):1111-1117. 